\title{
THE EFFECT OF AN OSCILLATING VERTICALLY ORIENTED MAGNETIC FIELD ON THE FERROFLUID LAYER LOCATED ON A PERFLUOROOCTANE SUBSTRATE
}

\author{
C.A. Khokhryakova (Bushueva), K.G. Kostarev, \\ A.V. Lebedev, M.O. Denisova \\ Institute of Continuous Media Mechanics of the Ural Branch, RAS, \\ 1 Ak. Korolev str., 614013 Perm, Russia
}

The paper presents the results of an experimental study of the deformation of a ferrofluid layer located on a liquid substrate exposed to a uniform vertically-oriented magnetic field, the amplitude of which varies in time according to a harmonic law. We restrict our study to the case of small amplitudes and frequencies, at which no ferrofluid layer disintegration takes place.

Introduction. A range of gravitational-capillary waves propagates along the free surface of an infinitely deep layer of fluid. Waves on the free surface of a magnetic fluid arise due to the fact that the pressure at any point of the fluid is proportional to the intensity in it. If the magnetic field changes periodically in time, periodic motions will be also excited on the free surface of magnetic fluid. This motion will be resonant in the collision of the characteristics of the surface wave excited by the magnetic field with temporal and spatial characteristics of the driving force [1]. The presence of a magnetic fluid surface (and interfacial) determines the influence of the spatial characteristics of the system, such as the diameter of the cuvette and the thickness of the liquid layer, on the resonant frequency.

The generation of surface waves in a magnetic fluid layer enhances the potential of laboratory modelling of wave processes. The use of a liquid substrate for a magnetic fluid layer also offers a great opportunity for the study of different types of equilibrium instability and fluid flow in such systems [2]. The critical intensity of themagnetic field sharply decreases in this case so that the fluid layer disintegrates into an ordered array of individual drops or polygonal structures which respond to changes in magnetic field intensity, frequency and direction [3].

In this paper, we report the results of an experimental investigation aimed at studying the behavior of a ferrofluid layer located on a liquid substrate exposed to an oscillating vertically-oriented magnetic field.

1. Experimental technique. Magnetite dispersed in kerosene and stabilized with oleic acid was used as a magnetic fluid $\left(\rho=1.4 \mathrm{~g} / \mathrm{cm}^{3}, \sigma=24.3\right.$ dyne $/ \mathrm{cm}$, $\left.M_{\mathrm{S}}=50 \mathrm{kA} / \mathrm{m}, \chi_{0}=5.2\right)$ in the experiment. Perfluorooctane was chosen as a liquid substrate $\left(\rho=1.7 \mathrm{~g} / \mathrm{cm}^{3}, \sigma=13.6\right.$ dyne $\left./ \mathrm{cm}\right)$.

A two-layered liquid system in the form of a short vertical glass cylinder with a diameter $D$ was placed in a cuvette. The thickness of the ferrofluid layer was defined as $h=m / \rho S$, where $m$ is the fluid mass, $S=\pi D^{2} / 4$ is the area of the layer, and $D$ is the inner diameter of the cuvette. The fluid mass and, accordingly, the layer thickness were determined by weighting a syringe with the ferrofluid on an electronic scales before and after ferrofluid injection into the cuvette. The thickness of the ferrofluid layer $h$ determined in this manner varied from 1 to $4 \mathrm{~mm}$. 


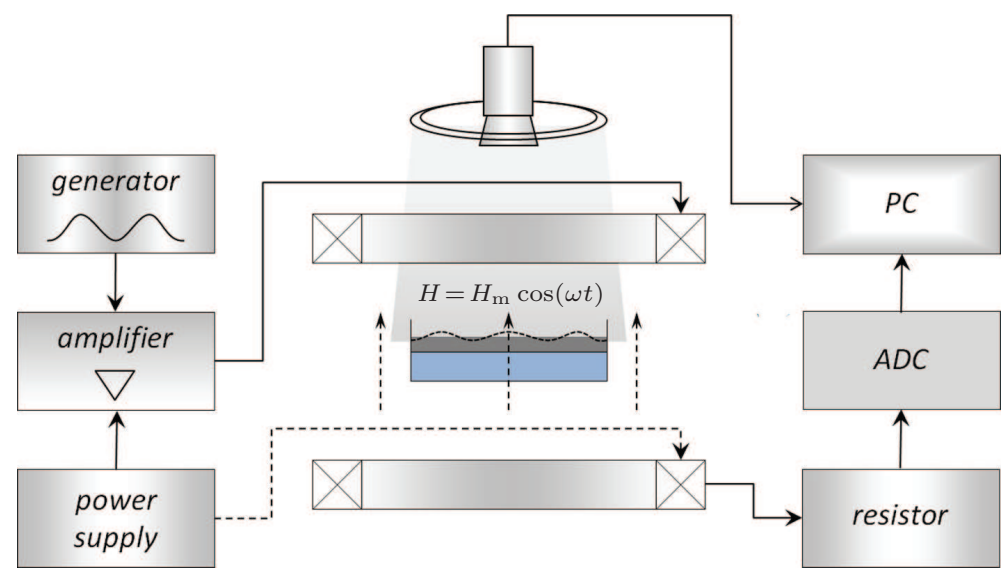

Fig. 1. Schematic of the experimental setup.

The thickness of the liquid substrate was several times greater than the thickness of the ferrofluid layer, varying, respectively, from 10 to $25 \mathrm{~mm}$.

In the course of the experiment, the two-layered liquid system (ferrofluid over perfluorooctane) in the cuvette [3] was placed on a horizontal pad in the central region of the Helmholtz coils (the uniformity of the magnetic field in this region $2 \%$ ). In order to initiate oscillations of the ferrofluid layer, an alternating magnetic field linearly polarized in a vertical plane was applied. To this end, the Helmholtz's coils were fed through an amplifier from a sinusoidal signal generator (a frequency range of $1-10 \mathrm{~Hz})$. A small resistor was connected in series to the coil, whose output signal was applied to the input of the ADC. The signal was processed using a standard software package. The image of the cell with a ferrofluid was taken by a digital video camera mounted above the system of the coils and equipped with a circular light source (Fig. 1).

Measurements were made under the quasi-stationary regime. As soon as the oscillation frequency of the magnetic field was changed, a picture of the surface relief was taken, but only after a pause that considerably exceeded the viscous time of the ferrofluid layer $(\tau=h D / 2 \nu$, where $\nu$ is the kinematic viscosity of the ferrofluid $\sim 15 \mathrm{cSt}$ ). The perturbations associated with the transient process faded during that time and only those, close to the proper wavelength of the system, remained. These perturbations were amplified by the magnetic field.

2. Experimental results. In the experiment, we applied an alternating linearly polarized magnetic field to the ferrofluid layer. The amplitude of the field was fixed and, with the given ferrofluid layer, was slightly lower than its critical strength $\left(H_{\mathrm{m}}=0.9 H_{\mathrm{c}}\right.$ compared to $H_{\mathrm{m}}=3.1 \mathrm{kA} / \mathrm{m}$ for a ferrofluid layer of the thickness $h=2.9 \mathrm{~mm}$ and $H_{\mathrm{m}}=3.7 \mathrm{kA} / \mathrm{m}$ for $h=3.3 \mathrm{~mm}$ ) at which the initially discontinuous layer disintegrated into separate drops [3].

In Fig. $2 a$, the top view of the horizontal ferrofluid layer under the action of the direct $(\nu=0 \mathrm{~Hz})$ magnetic field with the amplitude $H_{\mathrm{m}}=0.9 H_{\mathrm{c}}$ is shown. The free surface of the ferrofluid is the form of a pentagonal relief, which precedes the disintegration of the layer into an ordered system of drops [3]. The relief has its origin in the gravitational-capillary waves arising on the free surface of the layer in the absence of setup damping and enforced by the magnetic field. When apply an oscillating magnetic field, there is a competition between the gravity-capillary and the magneto-capillary perturbations generated by the ponderomotive force $F_{\mathrm{M}}$. These perturbations become predominant at the oscillating field frequency 
The effect of an oscillating vertically oriented magnetic field on the ferrofluid layer ...

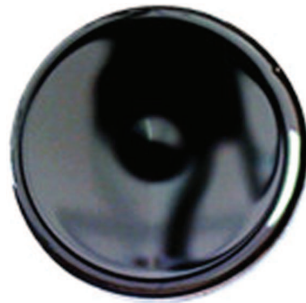

(a)

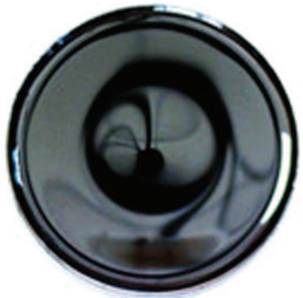

(b)

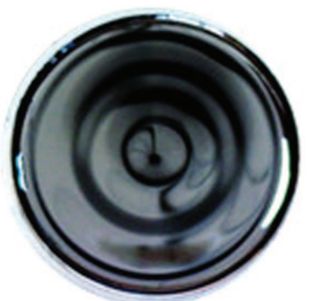

(c)

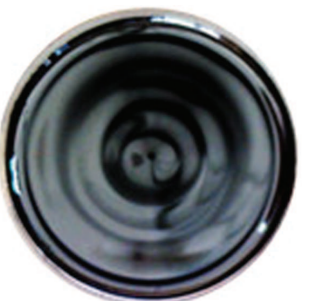

$(d)$

Fig. 2. Oscillations of the free surface of a ferrofluid layer on the liquid substrate at the magnetic field intensity amplitude $H_{\mathrm{m}}=0.9 H_{\mathrm{c}}$ and frequency $\nu, \mathrm{Hz}:(a)-0 ;(b)$ $-1 ;(c)-4 ;(d)-7$. The cuvette diameter $D=59.0 \mathrm{~mm}$, the ferrofluid layer thickness $h=3.3 \mathrm{~mm}$.

higher than $1 \mathrm{~Hz}$ and, therefore, the surface of the magnetic fluid layer undergoes periodical oscillations. Since the demagnetization factor of the ferrofluid layer in the near-wall zone is lower in comparison with the center of the cell (in view of the layer approximation by the plane), the meniscus is involved in the vibrational motion earlier than the rest of the layer. In addition, the tangential stresses caused by the field non-uniformity are distributed in the region near the cell boundary [4]. With this configuration, the oscillating meniscus turns out to be the source of waves travelling along the surface of the ferrofluid layer (Fig. 2b).

When the oscillation frequency of the external magnetic field increases, the travelling wave becomes a standing wave (see Figs. $2 c, d$ ). In particular, for a layer of $3 \mathrm{~mm}$ thick and $59 \mathrm{~mm}$ in diameter, this transition takes place at a frequency of the magnetic field intensity oscillation of $\sim 67 \mathrm{~Hz}$ which decreases with increasing liquid substrate thickness.

Fig. 3 illustrates the relationship between the wavelength of the surface waves $\lambda$ and the magnetic field frequency $\nu$, which is expressed as $\lambda=134 \nu^{-1.176}$. The dependence of the wavelengths of both travelling and standing waves on the field oscillation frequency is described by a single root law, and it is practically independent of the field strength amplitude. $\mathrm{T}$ he wavelength was determined as the spatial period of the wave process (as the distance between the hills and troughs, Fig. 3) which remained unchanged as the wave propagated from the periphery to the center of the cuvette.

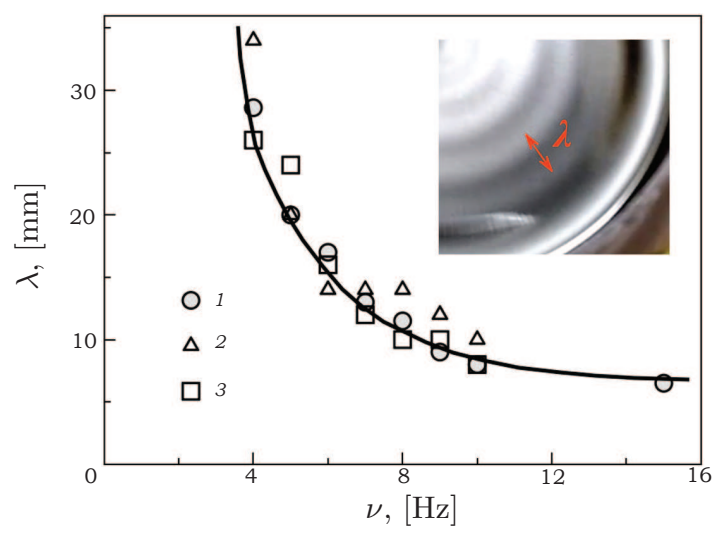

Fig. 3. The wavelength $\lambda$ on the ferrofluid layer free surface vs. the oscillation frequency of the driving force $\nu$ at the magnetic field intensity amplitude $H_{\mathrm{m}}: 1-0.9 H_{\mathrm{c}}$; $2-0.8 H_{\mathrm{c}} ; 3-0.75 H_{\mathrm{c}}$. 


\section{C.A. Khokhryakova,K.G. Kostarev, A.V. Lebedev, M.O. Denisova}

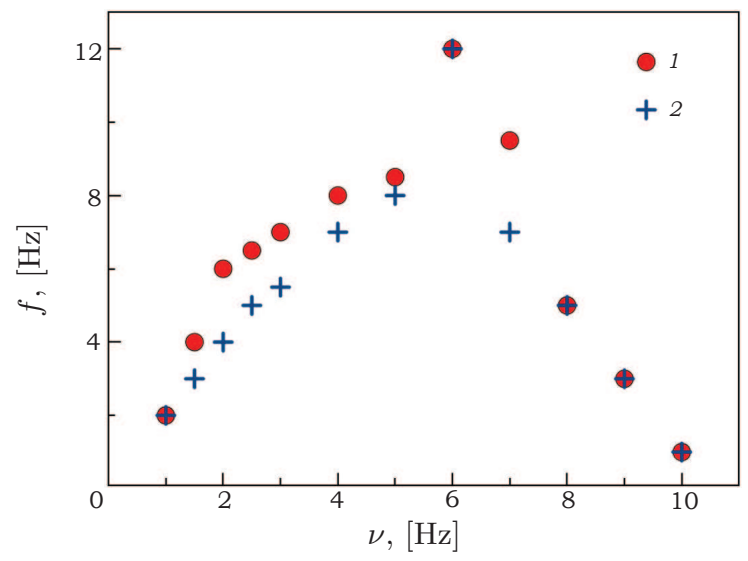

Fig. 4. The vibration frequency $f$ of the meniscus (1) and central part (2) of the ferrofluid layer free surface vs. the alternating magnetic field frequency $\nu$. The amplitude of magnetic field intensity oscillations $H_{\mathrm{m}}=0.9 H_{\mathrm{c}}$.
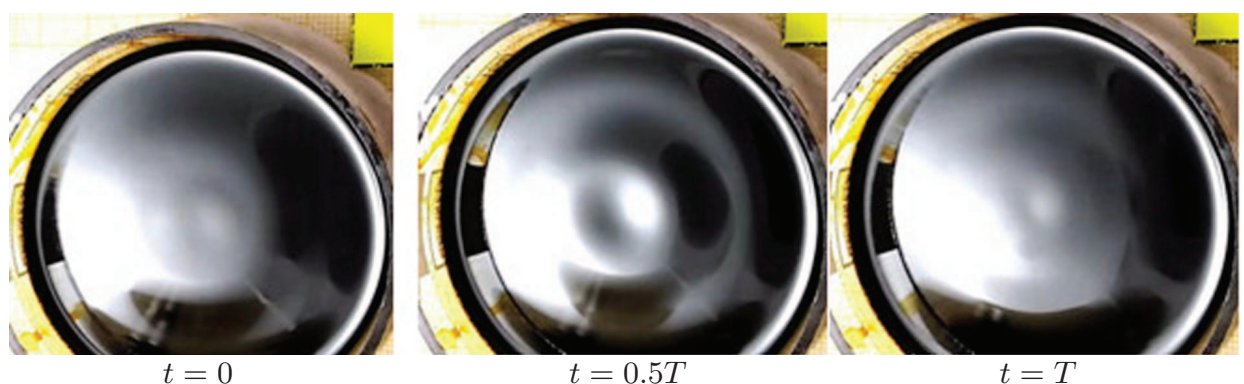

Fig. 5. Oscillations of the free surface of the ferrofluid layer of thickness $h=3 \mathrm{~mm}$ lying on a liquid substrate at the magnetic field strength amplitude $H_{\mathrm{m}}=0.9 H_{\mathrm{c}}$ and frequency $\nu=6 \mathrm{~Hz}$. The cuvette diameter $D=59.4 \mathrm{~mm}$.

By varying the frequency of the magnetic force oscillations at a fixed value of the magnetic field strength $H_{\mathrm{m}}=0.9 H_{\mathrm{c}}$, the frequency of the free surface vibrations of the magnetic fluid layer in the near-wall region (meniscus) and in the central part of the cuvette $(D=59 \mathrm{~mm})$ was investigated (Fig. 4). It has been found that the frequency of the meniscus oscillates monotonically with the frequency of the external driving force $\left(F_{\mathrm{M}} \sim 2 \nu\right)$ up to $6 \mathrm{~Hz}$, as well as the frequency of the layer oscillations in the central part which differs somewhat from the frequency of the meniscus because of the absence of contact with solid surfaces. After this, a sharp jump in dependence of $f(\nu)$ was observed when the travelling wave transformed into a standing wave. At the same time, the video surveillance system allowed us to observe and record the onset of the next oscillation mode of a frequency of $\sim 0.2 \mathrm{~Hz}$ on the ferrofluid layer free surface, resulting in periodic appearance (Fig. 5b) and disappearance (Figs. 5a,c) of a wave relief on the free surface of the ferrofluid layer. This effect can be attributed to the superposition of the characteristic wavelengths obtained for the free surface and interface of the ferrofluid layer.

The development of one or other oscillation mode of the ferrofluid surface is determined not only by the parameters of the external magnetic field, but also by the intrinsic geometric characteristics of the two-layer system, e.g., the cell diameter. In Fig. 6 , the oscillations of the free surface of the $3 \mathrm{~mm}$ thick ferrofluid 
The effect of an oscillating vertically oriented magnetic field on the ferrofluid layer ...

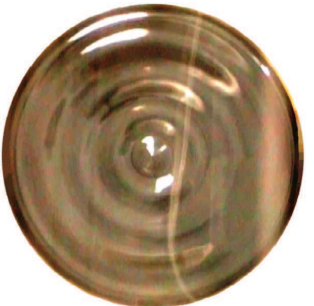

(a)

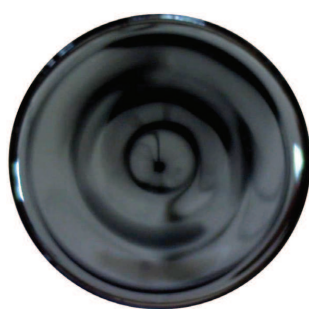

(b)

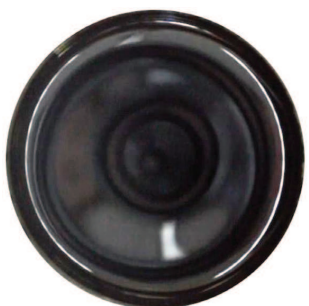

(c)

Fig. 6. Oscillations of the free surface of the ferrofluid layer under the action of the alternating magnetic field of amplitude $H_{\mathrm{m}}=0.9 H_{\mathrm{c}}$ and frequency $\nu=7 \mathrm{~Hz}$. The cuvette diameter $D$, mm: $(a)-89.0 ;(b)-59.4 ;(c)-35.0$.

layer in cuvettes of different diameters under the action of an alternating magnetic field with the amplitude $H_{\mathrm{m}}=1.9 \mathrm{kA} / \mathrm{m}$ and frequency $\nu=7 \mathrm{~Hz}$ are depicted. It is seen that the relief of the free surface of the layer and the dynamics of its change are essentially dependent on the diameter of the working cuvette.

3. Conclusions. The effect of an oscillating vertically-oriented magnetic field on a ferrofluid layer located on a liquid substrate was studied experimentally. It has been found that the wavelength of the excited standing wave decreases with increasing oscillation frequency of the magnetic field strength and increases as the cell diameter becomes larger. The results obtained in the study provide further insights into the evolution of multilayer systems, ferrofluids in the magnetic field and in the region of strong deformations and can be used to create a database for the verification of available mathematical models describing the behavior of a magnetic fluid layer with two free boundaries under the action of gravity.

Acknowledgements. The work was supported by RFBR project no. 1601-00517.

\section{References}

[1] B.M. Berkovsky, V.F. Medvedev and M.S. Krakov. Magnetic Fluids: Engineering Applications. (Oxford University Press, 1993).

[2] K.A. Bushueva and K.G. Kostarev. Behavior of a ferrofluid layer with stable surface rupture subjected to a tangential magnetic field. Fluid Dynam$i c s$, vol. 46 (2011), no. 5, pp. 707-714.

[3] C.A. Bushueva. Drop structures formed by ferrofluid in the uniform magnetic field. Magnetohydrodynamics, vol. 49 (2013), no. 3/4, pp. 598-602.

[4] A.V. LeBedev. Entrainment of a thin film of ferrocolloid by a rotating magnetic field. Magnetohydrodynamics, vol. 27 (1991), no. 4, pp. 461-462. 